University of Nebraska - Lincoln

DigitalCommons@University of Nebraska - Lincoln

$6-2001$

\title{
The role of interfaces in the magnetoresistance of $\mathrm{Au} / \mathrm{Fe} / \mathrm{Au} / \mathrm{Fe} /$ GaAs(001)
}

Axel Enders

University of Nebraska at Lincoln, a.enders@me.com

T. L. Monchesky

Simon-Fraser University

Kenneth Myrtle

Simon-Fraser University, myrtle@sfu.ca

R. Urban

Simon-Fraser University

Brett Heinrich

Simon-Fraser University, bheinric@sfu.ca

See next page for additional authors

Follow this and additional works at: https://digitalcommons.unl.edu/physicsenders

Part of the Physics Commons

Enders, Axel; Monchesky, T. L.; Myrtle, Kenneth; Urban, R.; Heinrich, Brett; Kirschner, Jurgen; Zhang, X.-G.; and Butler, W. H., "The role of interfaces in the magnetoresistance of $\mathrm{Au} / \mathrm{Fe} / \mathrm{Au} / \mathrm{Fe} / \mathrm{GaAs}(001)$ " (2001). Axel Enders Publications. 22.

https://digitalcommons.unl.edu/physicsenders/22

This Article is brought to you for free and open access by the Research Papers in Physics and Astronomy at DigitalCommons@University of Nebraska - Lincoln. It has been accepted for inclusion in Axel Enders Publications by an authorized administrator of DigitalCommons@University of Nebraska - Lincoln. 
Authors

Axel Enders, T. L. Monchesky, Kenneth Myrtle, R. Urban, Brett Heinrich, Jurgen Kirschner, X.-G. Zhang, and W. H. Butler

This article is available at DigitalCommons@University of Nebraska - Lincoln: https://digitalcommons.unl.edu/ physicsenders/22 


\title{
The role of interfaces in the magnetoresistance of $\mathrm{Au} / \mathrm{Fe} / \mathrm{Au} / \mathrm{Fe} / \mathrm{GaAs}(001)$
}

\author{
A. Enders, ${ }^{\text {a) }}$ T. L. Monchesky, K. Myrtle, R. Urban, and B. Heinrich \\ Simon-Fraser University, Physics Department, 8888 University Drive, Burnaby, BC, V5A 1S6, Canada \\ J. Kirschner \\ Max-Planck Institute, Weinberg 2, Halle, D-06120, Germany
}

X.-G. Zhang and W. H. Butler

Oak Ridge National Laboratory, P.O. Box 2008, Oak Ridge, Tennessee 37831-6118

\begin{abstract}
The electron transport and magnetoresistance (MR) were investigated in high quality crystalline epitaxial $\mathrm{Fe}(001)$ and $\mathrm{Au}(001)$ films and exchange coupled $\mathrm{Au} / \mathrm{Fe} / \mathrm{Au} / \mathrm{Fe} / \mathrm{GaAs}(001)$ trilayer structures. Fits to the experimental data were based on the semiclassical Boltzmann equation, which incorporates the electronic properties obtained from first-principles local density functional calculations. The fits require a surprisingly high asymmetry for the spin dependent electron lifetimes in $\mathrm{Fe}, \tau^{\downarrow} / \tau^{\uparrow}=10$ at room temperature. Despite the large atomic terraces at the Au/vacuum and $\mathrm{Fe} / \mathrm{GaAs}$ interfaces the scattering at the outer interfaces was found to be diffuse. The origin of MR in $\mathrm{Au} / \mathrm{Fe} / \mathrm{Au} / \mathrm{Fe} / \mathrm{GaAs}(001)$ structures is due to electron channeling in the Au spacer layer. The measured MR is consistent with the diffusivity parameters $s^{\uparrow}=0.55, s^{\downarrow}=0.77$ at the metal-metal interfaces. (C) 2001 American Institute of Physics. [DOI: 10.1063/1.1357844]
\end{abstract}

Although it is generally agreed that the origin of magnetoresistance (MR) is in spin dependent bulk and interface scattering, ${ }^{1}$ only little work has been done to test these ideas on well-defined crystalline epitaxial structures. In this work, the origin of the spin dependent scattering will be elucidated by modeling the measured MR of high quality crystalline $\mathrm{Au} / \mathrm{Fe} / \mathrm{Au} / \mathrm{Fe}$ epitaxial structures grown on semi-insulating $\operatorname{GaAs}(001)$ with the semiclassical Boltzmann equation that takes into account the electronic properties calculated from first principles.

The electron transport in the individual $\mathrm{Fe}(001)$ and $\mathrm{Au}(001)$ layers was investigated by in situ measurements of the sheet resistances in the current-in-plane geometry as a function of film thickness. The growth of the films was interrupted at certain thicknesses, and a head containing four $\mathrm{Be}-\mathrm{Cu}$ probes was lowered to one edge of the sample surface to measure the sheet resistance of the film using the van der Pauw geometry. ${ }^{2}$ The conductivity in the Fe films grown on $\operatorname{GaAs}(001)$ as a function of thickness is shown in Fig. 1(a). The experimental data were fitted with the semiclassical Boltzmann transport equation. ${ }^{3}$ The semiclassical Boltzmann equation used in this work employs realistic electronic band structure parameters, which are obtained from the firstprinciple calculations using the Layer Korringa-KohnRostoker method. The lifetimes of the majority and minority electrons, $\tau^{\uparrow}$ and $\tau^{\downarrow}$, as well as the specular scattering parameter $p$ at the surfaces were adjusted as free parameters to obtain the best fit to the experimental data. The results for $\tau^{\uparrow \downarrow}$ reproduce bulk conductivity for $\mathrm{Fe}$ at $300 \mathrm{~K}, \sigma_{\mathrm{Fe}}=0.1$ $\mu \Omega \mathrm{cm}^{-1}$. Surprisingly, the best fit to the data requires an unusually high ratio $\tau_{\downarrow} / \tau_{\uparrow}=10$ with $\tau_{\uparrow}=(2.5 \pm 1.2) \times 10^{-5}$ $\mathrm{s}, \tau_{\downarrow}=(3.0 \pm 0.2) \times 10^{-14} \mathrm{~s}$; and no specular scattering at the outer interfaces, $p=0$, and is shown as a solid line in Fig. $1(\mathrm{a})$.

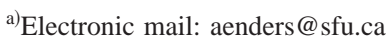

Using the Fermi velocities in $\mathrm{Fe}$ and the values for $\tau^{\uparrow}$, $\tau^{\downarrow}$ results in the mean free paths $\lambda^{\uparrow}=(11 \pm 6) \AA$ for majority electrons and $\lambda^{\downarrow}=(139 \pm 10) \AA$ for minority electrons. The use of any nonzero reflectivity at the outer interfaces, $p>0$, would require a higher asymmetry in the electron mean free path, e.g., for $p=0.25$ a significantly higher ratio $\tau_{\downarrow} / \tau_{\uparrow}$ $>17$ is required to reach an equivalent fit to the data. The error bar quoted for $\tau$ was deduced from the calculation of the $90 \%$ confidence interval of the fit. The large asymmetry of the electron lifetimes in $\mathrm{Fe}$ is not in agreement with that expected from arguments based on the density of states (DOS) of $d$ electrons given by Mott and co-workers. ${ }^{5}$ According to Mott, the ratio $\tau_{\downarrow} / \tau_{\uparrow}$ is expected to be inversely proportional to the ratio of the DOS of minority and majority $d$ electrons at the Fermi level. The first principle calculations give $\mathrm{DOS}^{\uparrow}=0.714 \mathrm{eV}^{-1}$ and $\mathrm{DOS}^{\downarrow}=0.272 \mathrm{eV}^{-1}$ for the majority and minority $d$ electrons. The ratio based on Mott's argument, $\tau_{\downarrow} / \tau_{\uparrow}=\operatorname{DOS}^{\uparrow} / \operatorname{DOS}^{\downarrow}=2.62$, results in the electron lifetimes $\tau^{\uparrow}=7.7 \times 10^{-15} \mathrm{~s}$ and $\tau^{\downarrow}=2.0 \times 10^{-14} \mathrm{~s}$ and yields the fit shown in Fig. 1(a) as a dotted line. Clearly, this fit is poor compared to that using the above unconstrained mean free path ratio $\tau^{\downarrow} / \tau^{\uparrow}=10$. The ratio $\lambda^{\downarrow} / \lambda^{\uparrow}=1.4$ obtained by Gurney and co-workers on sputtered $\mathrm{Fe}$ samples ${ }^{6}$ is much lower than that determined in this work. However, it is interesting to note that the mean free paths in Ref. 6 are close to the majority electron mean free path in this work. The mean free path in sputtered polycrystalline samples is strongly affected by structural defects and impurities. The role of scattering by defects would be most pronounced for minority electrons which have a large mean free path, $\lambda \downarrow$ $=(139 \pm 10) \AA$. The presence of scattering by defects in sputtered samples significantly decreases the minority electron mean free path and consequently results in a much lower $\tau_{\downarrow} / \tau_{\uparrow}$ ratio compared to that in good crystalline samples. Furthermore, the data points in Fig. 1 approach asymptotically the bulk conductivity in crystalline Fe, showing 


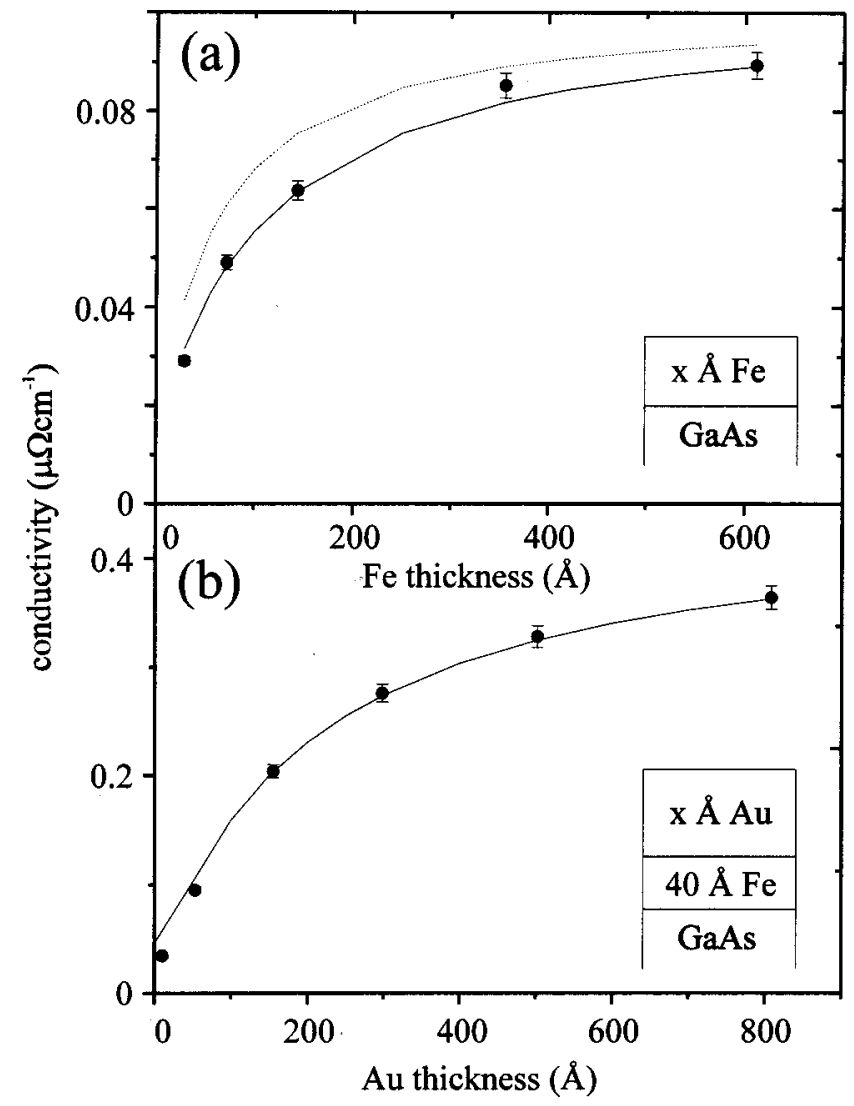

FIG. 1. Experimental data (circle) and first principles calculations of the Auand Fe-thickness dependence of the conductivity. (a) Fe/GaAs(001), solid: best fit with $\tau_{\downarrow}=(3.0 \pm 0.2) \times 10^{-14} \mathrm{~s}, \tau_{\uparrow}=(2.5 \pm 1.2) \times 10^{-15} \mathrm{~s}, p=0$; dashed: parameter based on ratio of $d$ DOS: $\tau^{\downarrow}=2.02 \times 10^{-14} \mathrm{~s}, \tau^{\uparrow}=7.71$ $\times 10^{-15} \mathrm{~s}, p=0$; (b) $\mathrm{Au} / 40 \AA \mathrm{Ae} / \mathrm{GaAs}(001)$, solid line: best fit given by the set of parameters described by line A in Fig. 2: $p=0, s^{\uparrow}=0.55, s^{\downarrow}=0.77$.

clearly the absence of impurity and defect scattering at room temperature (RT) in this samples.

To investigate the electron transport in $\mathrm{Au}(001)$ layers an additional set of experiments were carried out using $x$ $\mathrm{Au} / 40 \AA \mathrm{Fe} / \mathrm{GaAs}(001)$ structures, where $x$ was in the range of 0-800 $\AA$. The seed layer of Fe had to be used in order to get high quality crystalline Au layers. To improve the surface quality of the $\mathrm{Fe}$ template prior to the $\mathrm{Au}$ growth, the $\mathrm{Fe}$ growth was interrupted at a thickness of $28 \AA$ to remove surfactant As atoms by gentle sputter etching with $500 \mathrm{eV}$ $\mathrm{Ar}^{+}$ions under an angle of $75^{\circ}$ at RT. After sputtering, the sample was annealed at $200^{\circ} \mathrm{C}$, and an additional $12 \AA$ thick Fe layer was deposited at $200^{\circ} \mathrm{C}$. This preparation technique significantly enlarges the atomic terraces. ${ }^{7}$ The measured conductivities as a function of the Au thickness are shown as solid circles in Fig. 1(b). Again, the fit to these data was carried out by using the semiclassical Boltzmann equation. The electron lifetime in Au was set to $\tau^{\uparrow \downarrow}=2.77 \times 10^{-14} \mathrm{~s}$ $\left(\lambda^{\uparrow \downarrow}=380 \AA\right)$ to reproduce the bulk conductivity $\sigma_{\mathrm{Au}}$ $=0.440 \mu \mathrm{cm}^{-1}$ at RT. ${ }^{4}$ The fit parameters $s^{\uparrow}$ and $s^{\downarrow}$ were introduced to account for a partial diffuse scattering at the metal-metal interfaces. These parameters multiply the reflection $s \times R)$ and transmission $(s \times T)$ interface parameters. Since the $s^{\uparrow \downarrow}$ parameters strongly affect the $\mathrm{MR},{ }^{3}$ one has to use these parameters in fitting both the Au-thickness depen-

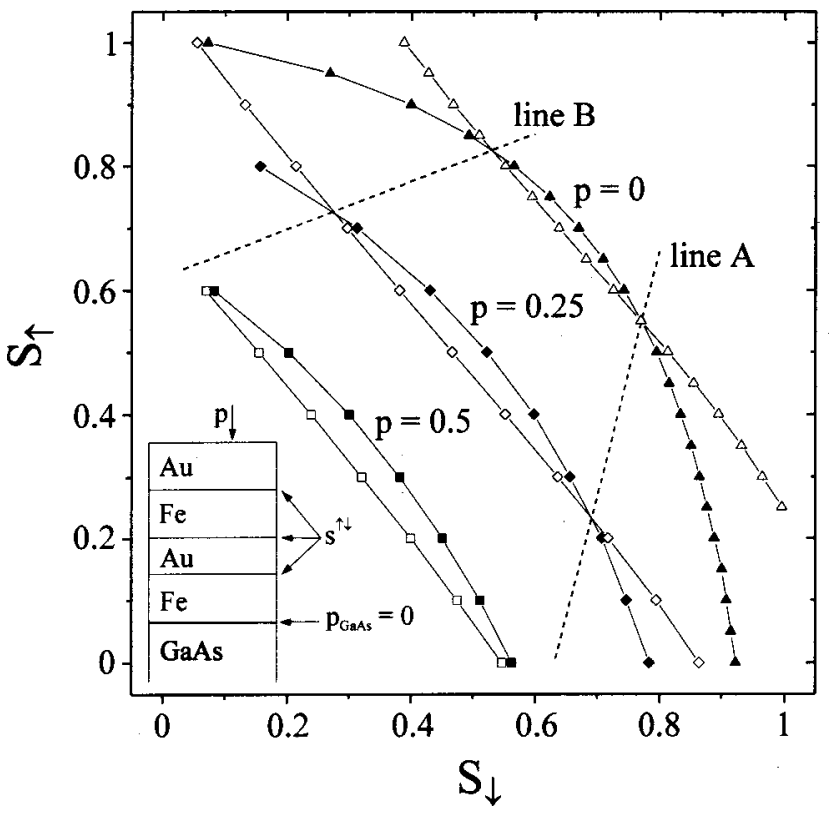

FIG. 2. Fitting of the measured room temperature conductivity of $20 \mathrm{ML}$ $\mathrm{Au} / 10 \mathrm{ML} \mathrm{Fe} / 7 \mathrm{ML} \mathrm{Au} / 28 \mathrm{ML} \mathrm{Fe} / \mathrm{GaAs}(001)$ : The filled symbols correspond to the set of $\left(s^{\uparrow}, s^{\downarrow}\right)$ which reproduce the measured conductivity for a parallel configuration of the magnetic moments in the Fe layers, and the open symbols for antiparallel alignment of the magnetic moments. Triangles, diamonds, and squares represent the calculations for $p=0,0.25$ and 0.5 . The dashed lines are interpolations of the points of intersection, which give the measured GMR ratio.

dence of the conductivity in $\mathrm{Au} / \mathrm{Fe}$ films and the measured $\mathrm{MR}$ in $\mathrm{Fe} / \mathrm{Au} / \mathrm{Fe}$ trilayer structures. The following approach is used: First, for a set of $p$ parameters $(p=0,0.25,0.5)$ the measured sheet resistances in $\mathrm{Au} / \mathrm{Fe} / \mathrm{Au} / \mathrm{Fe} / \mathrm{GaAs}(001)$ were modeled as a function of $s^{\uparrow}$ and $s^{\downarrow}$, as outlined in Fig. 2. The final choice of $p$ and corresponding $s$ parameters was then determined by fitting the Au-thickness dependence of the conductivity in $\mathrm{Au} / \mathrm{Fe} / \mathrm{GaAs}(001)$ samples.

The MR measurements were carried out ex situ on patterned $20 \quad \mathrm{ML} \quad \mathrm{Au} / 10 \quad \mathrm{ML} \quad \mathrm{Fe} / 7 \quad \mathrm{ML} \quad \mathrm{Au} / 28 \quad \mathrm{ML}$ $\mathrm{Fe} / \mathrm{GaAs}(001) .{ }^{8}$ For a $7 \mathrm{ML}$ thick Au-spacer layer the exchange coupling between the $\mathrm{Fe}$ layers is antiferromagnetic ${ }^{9}$ making it suitable for MR studies. The conductivities of $\sigma_{p}$ $=8.844 \mu \Omega \mathrm{m}$ and $\sigma_{a p}=8.692 \mu \Omega \mathrm{m}$ were measured in parallel and antiparallel alignment of the magnetic moments of the Fe layers at RT, giving a giant magneto resistance ratio (GMR) of $1.8 \%$. At $10 \mathrm{~K}$, the GMR changed to $2.9 \%$. The conductivity of the $\mathrm{Au} / \mathrm{Fe} / \mathrm{Au} / \mathrm{Fe}(001)$ structure was calculated as a function of $s^{\uparrow \downarrow}$ on each interface with $p_{\mathrm{GaAs}-\mathrm{Fe}}$ $=0$ and $p_{\text {Au-vac }}$ as a variable parameter, and by applying the bulk electron lifetimes as deduced earlier. The major problem for performing the fits is a large number of fit parameters, namely $2 n$ for a system consisting of $n$ metallic layers. In order to reduce the number of fit parameters, the same $s^{\uparrow \downarrow}$ have been assumed on each metallic interface. The lines along which the combination of $s^{\uparrow}$ and $s^{\downarrow}$ provides the measured conductivity in the $\mathrm{Au} / \mathrm{Fe} / \mathrm{Au} / \mathrm{Fe}(001)$ structure for parallel (solid symbols) and antiparallel (open symbols) configuration of the magnetic moments in the $\mathrm{Fe}$ layers are shown for $p_{\text {Au-vac }}=0$ (triangle), $p_{\text {Au-vac }}=0.25$ (diamond) and $p_{\text {Au-vac }}=0.5$ (square) in Fig. 2. The intersection of the two 


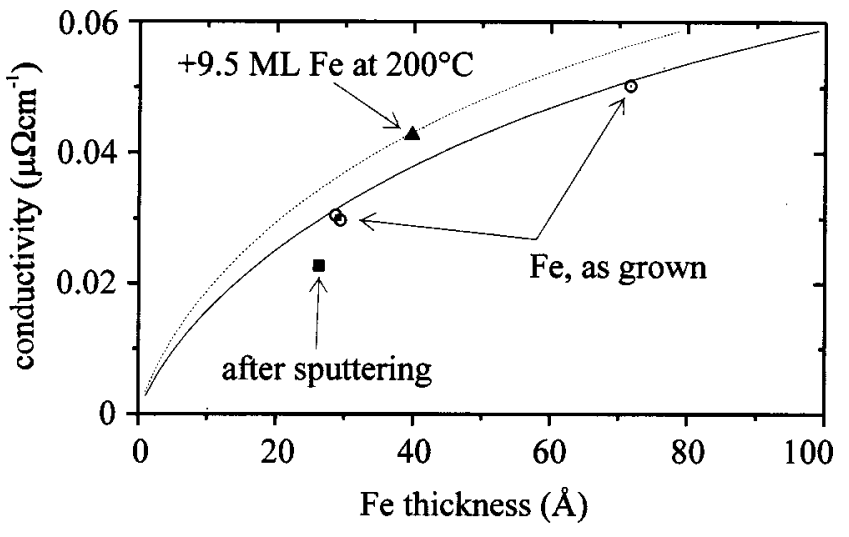

FIG. 3. Influence of the film preparation on the measured conductivity: with respect to the as-grown data (open circles), a rough film surface due to sputtering reduces the conductivity (square), while smoothening of the film surface due to elevated temperature growth increases the conductivity (triangle). Free electron fits to the data with $p=0$ (solid line) and $p=0.23$ (dotted line) for comparison.

corresponding lines for the parallel and antiparallel configuration of magnetic moments gives the solution with the right GMR. From now on, $p_{\text {Au-vac }}$ is simply referred to as $p$, since it is the only free specularity parameter at the outer interface, with $p_{\mathrm{GaAs}-\mathrm{Fe}}$ kept at zero as determined from the fits to the conductivity of $\mathrm{Fe}$ as a function of thickness. There are two solutions for each $p$. The interpolation of these solutions for the measured GMR as a function of $p$ is shown as dashed lines A and B in Fig. 2. In order to narrow down $p$ and $s^{\uparrow \downarrow}$, the fit quality parameter $\chi^{2}$ for the Au-thickness dependence of $\sigma$ was calculated with $p$ and $s^{\uparrow \downarrow}$ along lines A and B in Fig. 2 as fit parameters. The best fit was obtained for $p=0$ along both lines $\mathrm{A}$ and $\mathrm{B}$. The best fit for line A with $s^{\uparrow}=0.55, s^{\downarrow}=0.77$ and $p=0$ is shown in Fig. 1(b) as a solid line. It is equivalent to the best fit from line B with $s^{\uparrow}$ $=0.83, s^{\downarrow}=0.53$ and $p=0$ (not shown). This result of complete diffuse scattering of electrons at the outer $\mathrm{Au} /$ vacuum interface is surprising. A simple kinematical estimate based on the surface roughness measured directly by scanning tunneling microscopy (STM) (root-mean-square roughness of $\approx 1.1 \AA$ ) would result in $p>0$. Obviously, this simple kinematic picture does not apply here. The electron scattering even at relatively large interface atomic terraces with a width of $l=180 \AA$ cannot considered to be specular, one has to include the role of interface localized states and scattering processes caused by the atomic steps and defects in the surface reconstruction of $\mathrm{Au}(001)$ surface.

The preparation of the $\mathrm{Fe}$ seed layer for the $\mathrm{Au} / \mathrm{Fe} /$ $\mathrm{GaAs}(001)$ structures revealed the importance of surface smoothness on the specularity parameter $p$. The conductivities of Fe layers grown at RT are shown by open circles in Fig. 3. As discussed earlier, a complete diffuse scattering $p$ $=0$ is attributed to these data, and a fit based on a free electron model is shown as a solid line. The $25 \AA \mathrm{Fe}$ layer in Fig. 3 was sputtered by $500 \mathrm{eV} \mathrm{Ar}^{+}$ions to remove As surfactant atoms (solid square). The conductivity drop is attributed to damages created by $\mathrm{Ar}^{+}$ions within the $\mathrm{Fe}$ lattice. Subsequent annealing and the growth of $\mathrm{Fe}$ at $200{ }^{\circ} \mathrm{C}$ markedly improved the film conductivity (solid triangle). The growth at elevated temperatures shows a strongly increased magnitude in reflection high-energy electron diffraction oscillations. ${ }^{7}$ STM images give the terrace widths and the characteristic angles of roughness for the as-grown Fe film and annealed film to $45 \AA / 180 \AA$ and $9.6^{\circ} / 3.0^{\circ}$, respectively. This proves that the removal of As and subsequent annealing and growth at $200^{\circ} \mathrm{C}$ repaired the defects due to sputtering and improved the surface smoothness, which resulted in a higher film conductivity. The dotted line in Fig. 3 is a fit to the $\sigma$ of the annealed Fe film using the same mean free path as was employed for the solid line, but now the reflectivity at the $\mathrm{Fe} /$ vacuum interface was required to be increased to $p=0.23$. Therefore, a measurable degree in specular scattering was achieved by the growth at elevated temperatures.

A comparison between the measurements and first principles calculations allow now to plot the local conductance inside the $\mathrm{Au} / \mathrm{Fe} / \mathrm{Au} / \mathrm{Fe} / \mathrm{GaAs}(001)$ structure. The calculations showed that the GMR in these structures is due to high intrinsic reflectivity of both spin channels at the Fe/Au and $\mathrm{Au} / \mathrm{Fe}$ interfaces. The nature of this reflectivity is in a mismatch in electronic structures of the Fe and Au layers. GMR in this case is found to arise from multiple reflections within the Au spacer layer, known as spin dependent electron channeling. ${ }^{10}$ The spin asymmetry in $T$ and $R$ predicts a moderately high GMR of $10 \%$. However, the presence of a small degree of diffuse scattering at the metal interfaces damps the multiple scattering inside the Au spacer, which results in further decrease of GMR to the observed value of $1.8 \%$.

The authors would like to thank the Natural Sciences and Engineering Research Council of Canada for grants that supported this work. Two of the authors (A.E. and B.H.) would like to thank the Alexander-v.-Humboldt Foundation for generously supporting this work.

${ }^{1}$ S. S. P. Parkin, Phys. Rev. Lett. 71, 1641 (1993).

${ }^{2}$ L. J. van der Pauw, Philips Res. Rep. 13, 1 (1958).

${ }^{3}$ W. H. Butler, X.-G. Zhang, and J. M. MacLaren, J. Supercond. 13, 221 (2000)

${ }^{4}$ CRC Handbook of Chemistry and Physics, edited by D. R. Lide (CRC Press, Boca Raton, 1999).

${ }^{5}$ N. F. Mott and H. Jones, The Theory of the Properties of Metals and Alloys (Clarendon, Oxford, 1936).

${ }^{6}$ B. A. Gurney, V. S. Speriosu, J.-P. Nozieres, H. Lefakis, D. R. Wilhout, and O. U. Need, Phys. Rev. Lett. 71, 4023 (1993).

${ }^{7}$ T. L. Monchesky, B. Heinrich, R. Urban, K. Myrtle, M. Klaua, and J. Kirschner, Phys. Rev. B 60, 14 (1999).

${ }^{8}$ Patterning of the GMR structures was done in collaboration with Professor C. R. Bolognesi, School of Engineering Science, Simon Fraser University, Burnaby, BC, V5A 1S6, Canada.

${ }^{9}$ A. Fuß, S. Demokritov, P. Grünberg, and W. Zinn, J. Magn. Magn. Mater. 103, L221 (1992).

${ }^{10}$ W. H. Butler, X.-G. Zhang, D. M. C. Nicholson, T. C. Schulthess, and J. M. MacLaren, Phys. Rev. Lett. 76, 3216 (1996). 